\title{
ENT: Expanding Horizons
}

\section{Fefar AD*}

Department of ORL, PDU Medical College, India

*Corresponding author: Alpesh D Fefar, Assistant Professor, Department of ORL, PDU Medical College, Rajkot, India, Email: alpay.dr@gmail.com

\section{Editorial}

Volume 5 Issue 2

Received Date: August 10, 2020

Published Date: August 14, 2020

DOI: 10.23880 /ooaj-16000196

\section{Editorial}

ENT as a branch no longer has been removing wax, putting grafts or tonsillectomies. In last decade it has grown very fast. Refinement in the surgical techniques with availability of technical innovation has benefitted both surgeons and patients.

In otology, the concept has changed from hearing preservation to hearing augmentation. The use of TORP (Total Ossicular Reconstruction Prosthesis), PORP (Partial Ossicular Reconstruction Prosthesis), Piston have helped for better hearing outcome in tympanoplasties. Endoscopes in the ear gives surgeon full 3600 magnified view and made ear surgery minimal access surgery. Cochlear Implant has brought smile on many faces who are unlikely to benefit from anything along with Implants like BAHA, Bone bridge etc. Technically advanced hearing aids have helped patients hard of hearing with cure of tinnitus.

In Rhinology, The Endoscopes has made it possible to reach different crevices of skull and nose. The percentage of skull base surgeries done endoscopically is rising every day. With Image guided system, complication rate can be reduced. Dacryocystorhinostomy, optic and orbital decompression can be done through nose without leaving any external scars. As an ENT, we are now able to give cause of vertigo with the help of clinical examination and equipments like VEMP, ENG, VNG, Posturography etc. Sialo endoscopy makes direct visualization of tiny salivary gland ducts. Stroboscope has changed our image of diagnosing laryngeal lesions. The use of Microdebrider, Coblation, Laser has made surgeries stichless, Bloodless, precise. The latest in the field is robotic surgeries.

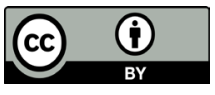

